\title{
Buckling Mode Transition in Composite Panels under Different Stiffening Conditions
}

\author{
Si-Yuan JIANG, Zhi-Dong GUAN* and Zeng-Shan LI \\ School of Aerospace Science and Engineering, Beihang University, \\ Beijing, 100191, China \\ *zdguan@buaa.edu.cn
}

Keywords: Composite, Stiffened panel, Post-buckling, Buckling mode transition.

\begin{abstract}
Two stiffened composite panel configurations which were designed with the same skin but different $\mathrm{T}$-shape stiffeners were took into consideration and loaded in uniaxial compression to collapse. Comparison were made in terms of buckling mode, structural stiffness and failure mode. Scale factors of the first and second modes were predicted employing a deduced energy method, and were implemented in the post-buckling analysis, which was performed in a commercial FEM code ABAQUS. Experimentally and numerically, buckling mode transition was observed merely in the panel with lighter stiffeners. A good agreement between simulation and test validates the prediction method and indicates that a higher stiffener stiffness restrains the transition of buckling mode, which may cause the instability of structural stiffness loaded in uniaxial compression.
\end{abstract}

\section{Introduction}

Stiffened composite panel construction is characterized by a skin and longitudinal stiffeners across the panel width, and is capable of supporting compressive load in excess of the critical buckling load, called post-buckling behavior. [1],[2] After initial buckling of the structure, the skin between the stiffeners presents different buckling modes, which can be distinguished by their wave numbers and cause redistribution of stress.[3],[4] For some configurations of such structure, more than one mode can be observed, which indicates there is a transition of buckling mode in the process of post-buckling. The transition, is often referred to as a mode-switch, mode-jump or modechange, results in a load transformation in mechanisms and failure mode.

Much experiment and research have focused on this phenomenon and the structure's postbuckling behavior. Stoll et al.[5] were among the first to demonstrate the initial buckling and modetransition of a metallic plate in uniaxial compression with a FEM method. Shin et al. expounded the reason of the transition using a Marguerre-type energy method, based on an experiment with an anisotropic composite panel.[6] The magnitudes of the total potential energy for deformed shapes associated with different buckling modes were employed to predict the transition. Falzon et al. developed a modified explicit procedure which could predict the mode-transition with better accuracy and more efficient than standard explicit dynamic analysis.[7],[8] And Wadee developed a nonlinear analytical model to predict the interaction between the global buckling mode and local buckling mode.[9]

On the post-buckling behavior, Falzon and Orifici et al. demonstrated how buckling mode affect the failure mode and the nodal/anti-nodal line are critical position of the failure.[10], [11] Different FEM methods were compared by Lanzi et al.[12] to strengthen Falzon's theory and a "global-local" analysis technique was developed by Kong el al. for rapid evaluation on post-buckling behavior.[13],[14]

This paper presents the results of an experimental study of two composite panels stiffened with different blades and numerical analysis. Also presented are the results of a deduced energy method which is employed to calculate factors of the first and second mode. 


\section{Experiment}

\section{Test Specimens}

Key dimensions of the specimens are shown in Fig. 1. The panels shared the same skin but differs in height of the T-shape stiffeners, and were manufactured in the same co-cured process.

To ensure the uniform loading and prevent premature failure at loading ends, the upper and lower ends of the panels were reinforced by epoxy resin blocks with steel frames. The stacking sequence of both the skin and the stiffeners are $\left[ \pm 45 / 0_{3} / 45 / 90 /-45_{2} / 0_{2} / 45 / 0_{2} / 45 / 90 /-45 / \overline{0}\right]_{s}$, while the heights of the stiffeners for specimen I and II are $35 \mathrm{~mm}$ and $40 \mathrm{~mm}$, separately.

All layers were composed of the CCF800/AC531 unidirectional tape whose thickness was 0.137 mm except the \pm 45 layer was CF8081/AC531 fabric whose thickness was $0.185 \mathrm{~mm}$. All the mechanical properties are listed in Table 1.

Table 1 Material properties

\begin{tabular}{llllllll}
\hline Material & $\mathrm{E}_{11} / \mathrm{GPa}$ & $\mathrm{E}_{22} / \mathrm{GPa}$ & $\mathrm{G}_{12} / \mathrm{GPa}$ & $\mathrm{X}^{\mathrm{T}} / \mathrm{MPa}$ & $\mathrm{X}^{\mathrm{C}} / \mathrm{MPa}$ & $\mathrm{Y}^{\mathrm{T}} / \mathrm{MPa}$ & $\mathrm{Y}^{\mathrm{C}} / \mathrm{MPa}$ \\
\hline Unidirectional tape & 155 & 10.1 & 3.9 & 2374 & 1158 & 32.6 & 220 \\
\hline Fabric & 73.9 & 73.9 & 3.9 & 900 & 671 & 900 & 661 \\
\hline
\end{tabular}

Note: $E_{11}$ represents longitudinal modulus; $E_{22}$ represents transverse modulus; $G_{12}$ represents inplane shear modulus; $\mathrm{X}$ represents longitudinal strength; Y represents transverse strength; Subscript T represents tension; Subscript $\mathrm{C}$ represents compression.

\section{Test Set-up}

The uniaxial compression tests of the stiffened composite panels have been performed with a hydraulic test machine WAW-2000A, whose maximum design capability is $2000 \mathrm{kN}$. The specimens were loaded through controlling the displacement of the loading end by the speed of $1 \mathrm{~mm} / \mathrm{min}$. The strain gauges were located on the skin through the waves in the first and second buckling modes of the stiffened panel as shown in Fig. 1, which were determined by a finite element model developed in ABAQUS/Standard. For observing the buckling mode in the experiment, 14 strain gauges (labeled A1-A7, B1-B7) were bonded back-to-back on both sides of each skin bay among the stiffeners, monitoring the out-of-plane deformation during the loading process. Among them, the letter A refers to the gauges on the side with stiffeners while B refers to the plane side. Simply support was set at $225 \mathrm{~mm}$ upper and lower to the axial center line.

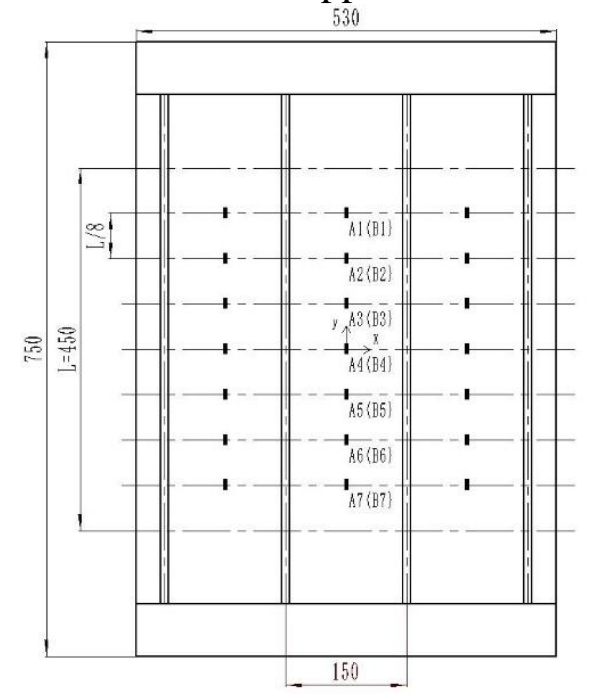

Fig. 1Schematic of the composite stiffened panels and distribution of strain gauges 


\section{Test Results}

As shown in Fig. 2, the data obtained from the gauges indicate an obvious buckle phenomenon in the experiment, which can be easily observed according to the point where the slopes of strain-load curves for back-to-back strain gauges on the skin change suddenly into two opposite direction. Apparently, the strain-load curves are quite close to linear till the initial buckling load. The critical load of panel I and II is $685 \mathrm{kN}$ and $710 \mathrm{kN}$, respectively. However, the difference between A9 and B9 of panel I reversed when the load reached $880 \mathrm{kN}$, which indicated the mode of out-ofplane deformation changed at this point, and buckling mode of the structure transited into a new mode. The gradual reduction in the rate of bending strain shown in Fig. 2(a) as a function of compression load, was due to the transition to a higher mode shape causing a node-line shift.
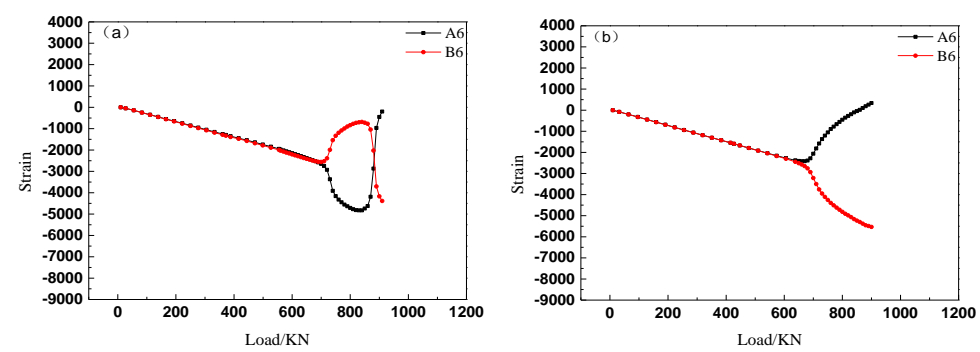

Fig. 2Typical strain-load curve obtained on certain location

(a) results of panel I ; (b)results of panel II

Fig. 3 shows the bending strain of different locations in one skin bay under a series of compression loading, which indicates the number of buckling mode half-waves directly. Obviously, the buckling mode of panel I was initially characterized by a shape of 2 half-waves, and switched to a 3 half-waves mode as the load increased. While the buckling mode stayed stable as a 2 halfwaves shape in panel II till collapse. The load-displacement curves are shown in Fig. 4. Curves of the two panels kept linear before the initial buckling and suffered a reduction of rate after it. For panel II, the curve changes into a new linear stage, and the stiffness keeps stable till collapse. Yet the stiffness of panel I varies continuously, and is not able to keep stable until the mode transition is done.
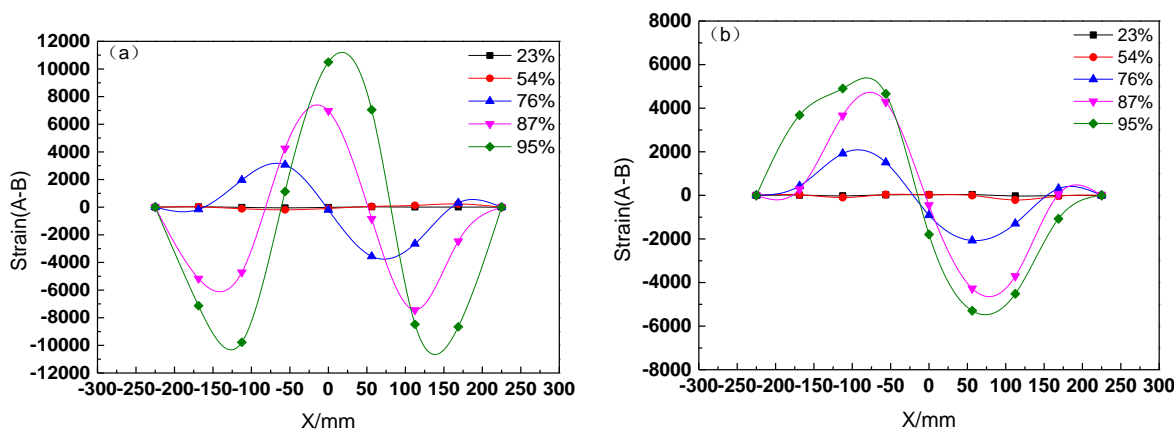

Fig. 3Difference of strain between A and B obtained on various locations under load sequence

(a) results of panel I ; (b) results of panel II

The collapse of the two panels happened in a flash along the horizontal line, accompanied with a compression load drop and complex post-buckling failure modes including fiber fracture, delamination, matrix crack and dramatic out-of-plane deformation. Obviously, the failure positions of the panels are different from each other (Fig. 5). Panel I failed at the middle height of the panel, while panel II failed at one quarter length from the top support edge.

Different test phenomenon shows the effect of the stiffener to the buckling modes, stiffness and failure mode of stiffened composite panels. A heavier stiffener is liable to restrain the transition of buckling mode and keep the stiffness stable. 


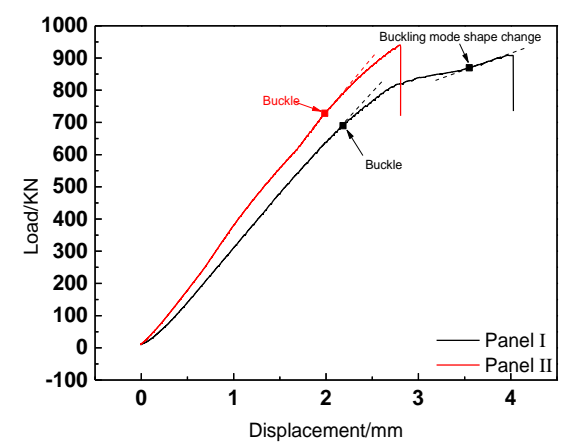

Fig. 4Load-displacement curve of the panels

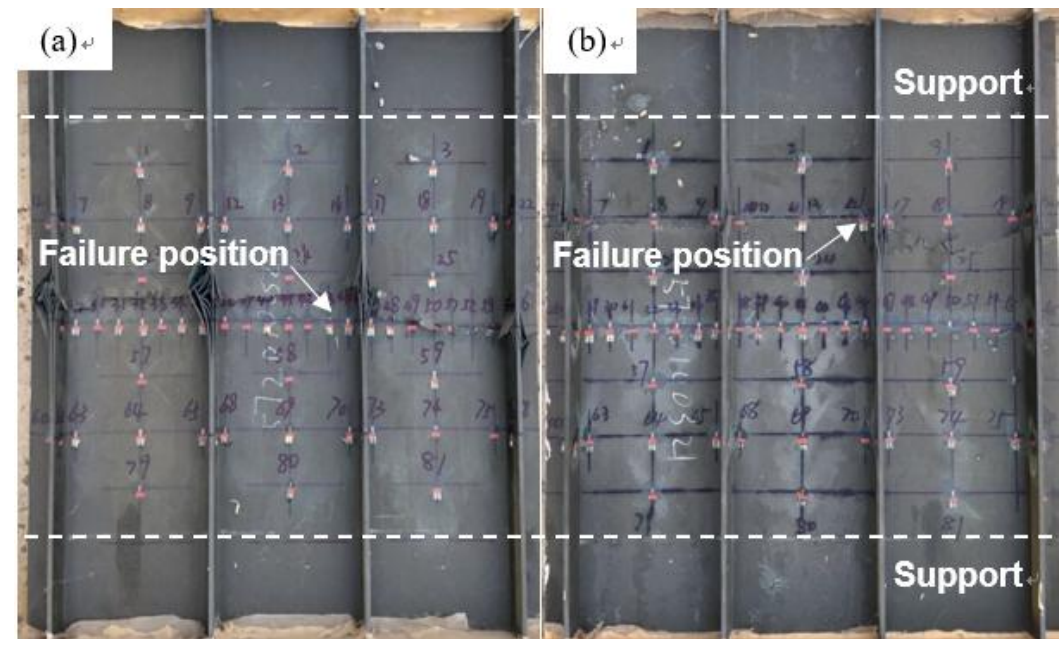

Fig. 5Failure mode of panels

(a)panel I ; (b)panel II

\section{Numerical analysis}

\section{FEM Models}

Finite element analyses are performed using the commercial code, ABAQUS, version 6.13.1. Stiffened panels are modeled by 8-nodes shells SC8R. After a preliminary mesh sensitivity study, the dimensions of shell elements are selected equal to $8 * 8$. A linear elastic behavior is considered as constitutive material law. Restrain all the degrees of freedom of the top and bottom edges of the panel expect an axial movement of one edge.

\section{Eigenvalue Analysis}

Eigenvalue analysis is used to evaluate the initial buckling load and to learn the buckling mode shapes(Fig. 6). The results show that the initial buckling mode of two panels is characterized by a 2 half-waves deformation, and corresponding load for the two panels are $669 \mathrm{kN}$ and $698 \mathrm{kN}$, respectively. While the load associated with the second mode ( 3 half-waves) is $681 \mathrm{kN}$ and $722 \mathrm{kN}$, which is quite close to them of the first mode. The proximity of the buckling loads indicates the sensitivity of the buckling mode to compressive load for this structure. 
(a)

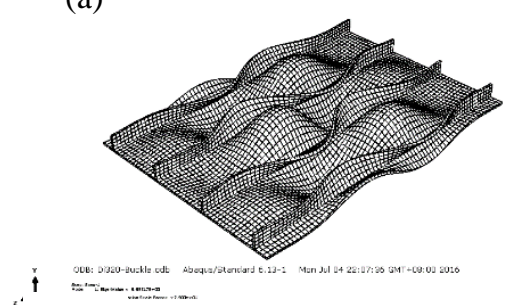

(b)

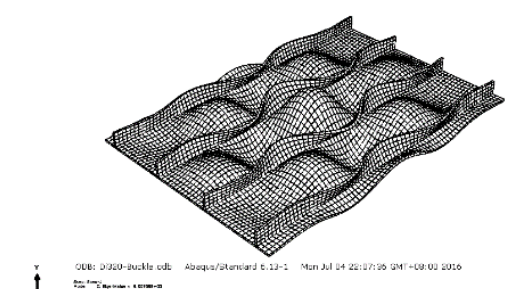

主

Fig. 6Mode shapes obtained from eigenvalue analysis

(a) the first mode; (b) the second mode

\section{Scale Factors}

A deduced energy method was employed to determine the scale factors of the first and second mode before the FEM post-buckling analysis. The procedure is based on Shin's energy method[6] and simplified with Falzon's theory[15].

One skin-bay between two stiffeners is selected as the object of study and the stiffeners are considered as boundary conditions. The stiffeners did not provide complete restraint against inplane movement, so an upper and a lower limit can be obtained when the stiffeners are considered as idealized boundary conditions. The first condition allowing in-plane movement of the unloaded edges (BC1) while the second had fully restrained unloaded edges (BC2).

The general compatibility equation for large deflection governing the post-buckling behavior of orthotropic plates can be expressed as

$$
A_{22}^{*} \Phi_{, x x x}+\left(2 A_{12}^{*}+A_{66}^{*}\right) \Phi_{, x x y y}+A_{11}^{*} \Phi_{, y y y y}=w_{, x y}^{2}-w_{, x x} w_{, y y},
$$

where ${ }^{A_{i j}}$ were membrane stiffness matrix terms, $\Phi$ is a stress function and $\mathrm{w}$ is the function of out-of-plane displacement, which is assumed as

$$
\mathrm{w}=W_{21} \sin \frac{2 \pi x}{a} \sin \frac{\pi y}{b}+W_{31} \sin \frac{3 \pi x}{a} \sin \frac{\pi y}{b},
$$

where a/b are length/width of the skin bay, $W_{21}$ and $W_{31}$ are unknown amplitudes.

Eq. (2) is selected as the deflection not only because it fits the boundary conditions, but also ensures that the plate can deform into 2 half-waves, 3 half-waves mode or a mode which is the superposition of them, depending on the unknown parameters $W_{21}$ and $W_{31}$. By investigating them, the scale factors of first and second mode can be determined.

The solution of Eq. (1) consists of a complementary part can a particular part. And a complementary solution can be obtained as

$$
\Phi c=-\frac{1}{2}\left(\widehat{N}_{y} x^{2}+\widehat{N}_{x} y^{2}\right)
$$

where $\widehat{N}_{x}$ and $\widehat{N}_{y}$ are averaged in-plane loads in the $\mathrm{x}$ and y directions, respectively.

From the classic laminate theory, the displacement in the $\mathrm{x}$ and $\mathrm{y}$ directions can be expressed as 


$$
\begin{aligned}
& \Delta_{x}=-\int_{0}^{a}\left(A_{11}^{*} \Phi_{, y y}+A_{12}^{*} \Phi_{, x x}-\frac{1}{2} w_{, x}^{2}\right) d x, \\
& \Delta_{y}=-\int_{0}^{b}\left(A_{12}^{*} \Phi_{, y y}+A_{22}^{*} \Phi_{, x x}-\frac{1}{2} w_{, y}^{2}\right) d y .
\end{aligned}
$$

By substituting $\mathrm{w}$ and $\Phi$, these displacements were obtained in terms of $\widehat{N_{x}}, \widehat{N_{y}}, W_{21}, W_{31}$ :

$$
\begin{aligned}
& \Delta_{x}=f\left(\widehat{N_{x}}, \widehat{N_{y}}, W_{21}, W_{31}\right), \\
& \Delta_{y}=g\left(\widehat{N_{x}}, \widehat{N_{y}}, W_{21}, W_{31}\right) .
\end{aligned}
$$

When appropriately manipulated, $\widehat{N}_{x}$ and $\widehat{N}_{y}$ were expressed as:

$$
\begin{aligned}
& \widehat{N_{x}}=f^{\prime}\left(\Delta_{x}, \Delta_{y}, W_{21}, W_{31}\right), \\
& \widehat{N_{y}}=g^{\prime}\left(\Delta_{x}, \Delta_{y}, W_{21}, W_{31}\right) .
\end{aligned}
$$

Expressions for the amplitudes $W_{21}$ and $W_{31}$ were next derived by the principle of minimum potential energy. The derivation of the strain energy can be expressed as:

$$
\left.\Pi=\frac{1}{2} \iint_{S}^{\left(A_{11}^{*} \Phi_{, y y}^{2}+2 A_{12}^{*} \Phi_{, x x} \Phi_{, y y}+A_{22}^{*} \Phi_{, x x}^{2}+A_{66}^{*} \Phi_{, x y}^{2}+D_{11} w_{, x x}^{2}+\right.} D_{12} w_{, x x} w_{, y y}+D_{22} w_{, y y}^{2}+4 D_{16} w_{, x x} w_{, x y}+4 D_{26} w_{, y y} w_{, x y}+4 D_{66} w_{, x y}^{2}\right) d S^{.} .
$$

Thus, the potential energy $\Pi$ was obtained only in terms of $\Delta_{x}, \Delta_{y}, W_{21}, W_{31}$.

When the plate buckles with a boundary condition of BC1, the average load $\widehat{N}_{y}$ is zero, while when it buckles with BC2, ${ }^{\Delta_{y}}$ is zero. Thus ${ }^{\Delta_{y}}$ can be expressed only in terms of $\Delta_{x}, W_{21}$ and $W_{31}$ by Eq. (6).

The unknown amplitudes were then solved for by setting up the following equations for minimum potential energy

$$
\begin{aligned}
& \partial \Pi\left(\Delta_{x}, W_{21}, W_{31}\right) / \partial W_{21}=0, \\
& \partial \Pi\left(\Delta_{x}, W_{21}, W_{31}\right) / \partial W_{31}=0 .
\end{aligned}
$$

All the procedures were coded in MATLAB, and a result of the ratio $W_{21} / W_{31}$ was obtained as shown in Fig. 7. The ratio boosts rapidly at first and reaches a plateau as the end-shortening $\Delta_{x}$ increases. Obviously, the upper limit (BC1) of the ratio is 1.40 while the lower limit (BC2) is 1.35.

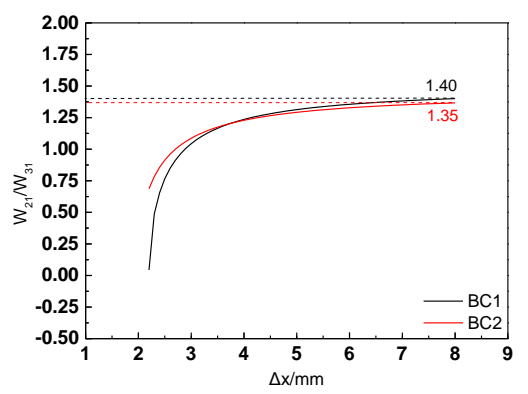

Fig. 7Result of scale factors calculation with different boundary conditions 


\section{Post-buckling Analysis}

To consider post-buckling behavior of the structure, an imperfection combined with the first and second mode was superimposed on the initial geometry of the FEM model. The imperfection was obtained by:

$$
\Delta x=\sum_{i=1}^{2} w_{i} \phi_{i}
$$

where $\phi_{i}$ is the $\mathrm{i}^{\text {th }}$ mode eigenvector from the result of previous eigenvalue analysis, and $w_{i}$ is the scale factor of the $i^{\text {th }}$ mode.

$5 \%$ of the skin-thickness was set as scale factor of the first mode. ${ }^{[7]}$ Based on the conclusion of section 3.3 and a series of sensitivity study, scale factor of the second mode was set as $3.65 \%$ of the thickness.

An explicit dynamic analysis was performed using ABAQUS/Explicit. The same mesh number, constraints and material characteristics are used as the eigenvalue analysis. An obvious transition of buckling mode was presented in the analysis of panel I . As shown in Fig. 8, at 50\% of the collapse load, 2 half-waves buckling mode is presented, while the mode switches into 3 half-waves at $85 \%$. Comparison is made when the buckling mode of panel II features stable as 2 half-waves till collapse as the result shows.

By investigating the load-displacement curve(Fig. 9) which is given by the analysis, the curve of panel I stays linear expect a fluctuation between $710 \mathrm{kN}$ and $900 \mathrm{kN}$, which are only $3.6 \%$ and $2.3 \%$ higher than the initial buckling and mode-transition loads in experiment, while no such phenomenon in panel II. There is only a reduction of stiffness found in panel II, which happens at the load of $690 \mathrm{kN}$. Since Hashin's two-dimensional failure criterion was introduced in the analysis, failure load and location can be predicted for each structure. Two curves drop dramatically at a load of $944 \mathrm{kN}$ and $995 \mathrm{kN}$ respectively, which indicate collapse of the structures. Besides, failure mode prediction shows that the anti-nodal lines were the critical position of failure, which is caused by the tremendous out-of-plane displacement. Thus, failure position for panel I is the axial 1/2 or 1/4 line of the panel I, while only the 1/4 line for panel II (Fig. 10).

All predictions of the numerous analysis accord well with the experiment.
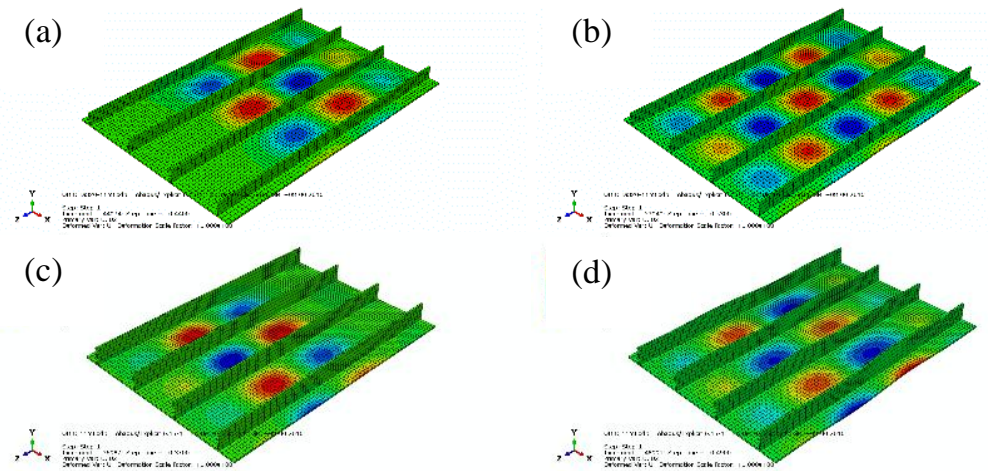

Fig. 8Simulation of out-of-plane displacement under different loads

(a)panel I 50\% collapse load; (b)panel I 85\% collapse load;

(c)panel II 50\% collapse load; (d)panel II 85\% collapse load 


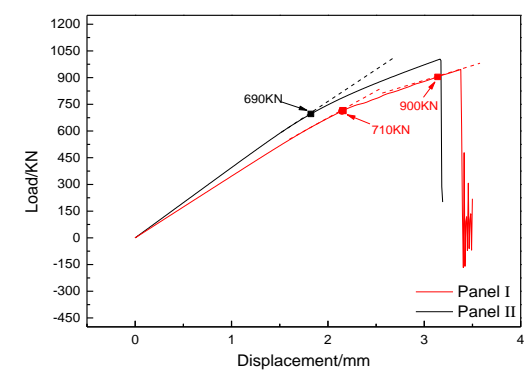

Fig. 9Load-displacement curves of numerous result

Based on the classic laminate theory

$$
\left[\begin{array}{l}
\sigma_{x} \\
\sigma_{y} \\
\sigma_{x y}
\end{array}\right]=\left[\begin{array}{ccc}
A_{11} & A_{12} & 0 \\
A_{12} & A_{22} & 0 \\
0 & 0 & A_{66}
\end{array}\right]\left[\begin{array}{c}
\varepsilon_{x} \\
\varepsilon_{y} \\
\varepsilon_{x y}
\end{array}\right],
$$

equivalent stiffness of the stiffeners can be obtained as

$$
(E A)_{x}=\left(A_{11}-A_{12}^{2} / A_{22}\right) b .
$$

Since the material properties and layers for the stiffeners of panel I and II are all the same, the ratio of stiffness for stiffeners I and II is determined by b, the height of the stiffeners. As the height of stiffeners for panel I is designed $12.5 \%$ less than panel II, the different performance of them ban be explained by this sole difference.

(a)
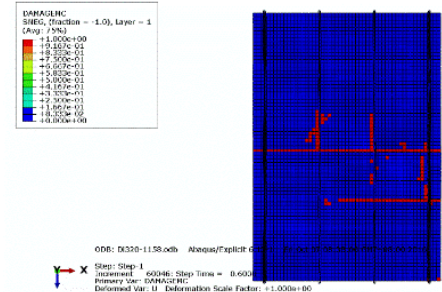

(b)

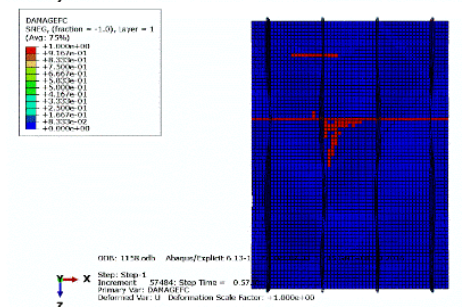

Fig. 10Simulation of failure mode

(a) result of panel I ; (b)result of panel II

\section{Conclusions}

Two T-stiffened composite panels were tested to failure. Both buckled into a 2 half-waves mode at $685 \mathrm{kN}$ and $710 \mathrm{kN}$, while the panel with lighter stiffeners suffered a transition into 3 half-waves mode at $880 \mathrm{kN}$. The scale factors determined by the deduced energy method help the simulation of buckling mode transition when implemented in FEM post-buckling analysis. A good agreement with experiment and simulation in terms of the stiffness of structures and failure mode was obtained. Results show that when compressively loaded, stronger stiffeners are liable to restrain the transition of buckling mode, which fluctuate the stiffness of the whole structure. And the anti-nodal lines are vulnerable to suffer from failure. 


\section{References}

[1] Kong Bin, Chen Puhui, Chen Yan. Post-buckling failure evaluation method of integrated composite stiffened panels under uniaxial compression, J. Acta Materiae Compositae Sinica. 31,765(2014).

[2] Falzon B G, Cerini M. An automated hybrid procedure for capturing mode-jumping in postbuckling composite stiffened structures, J. Composite Structures, 73,186(2006).

[3] Zimmermann R, Masiero E, Ghilai G, et al. Improved Postbuckling Simulation for Design of Fibre Composite Fuselage Structures - The POSICOSS Project, C. Israel Conference on Aerospace Sciences, Tel Aviv40(2004).

[4] Chen Guijuan, Jiao Guiqiong, Xiong Wei. Nonlinear postbuckling behavior of composite laminate plates, J. Journal of Wuhan University of Science \& Technology: Natural Science Edition. 28,103(2005).

[5] Stoll F, Olson S, Stoll F, et al. Finite element investigation of the snap phenomenon in buckled plates, C Structures, Structural Dynamics, and Materials Conference(1997).

[6] Dong K S, Jr O H G, Gürdal Z. Postbuckling response of laminated plates under uniaxial compression, J. International Journal of Non-Linear Mechanics.28,95(1993).

[7] Falzon B G, Hitchings D. Capturing mode-switching in postbuckling composite panels using a modified explicit procedure, J. Composite Structures.60,447(2003).

[8] Falzon B G, Cerini M. An automated hybrid procedure for capturing mode-jumping in postbuckling composite stiffened structures, J. Composite Structures. 73,186(2006).

[9] Wadee M A, Farsi M. Local-global mode interaction in stringer-stiffened plates, J. ThinWalled Structures. 85,419(2014).

[10]Falzon B G, Stevens K A, Davies G O. Postbuckling behaviour of a blade-stiffened composite panel loaded in uniaxial compression, J. Composites Part A Applied Science \& Manufacturing. $31,459(2000)$.

[11] Orifici A C, Shah S A, Herszberg I, et al. Failure analysis in postbuckled composite T-sections, J. Composite Structures. 86,146(2008).

[12]Lanzi L, Bisagni C. Post-Buckling Experimental Tests and Numerical Analyses on Composite Stiffened Panels, C. Structures, Structural Dynamics, and Materials Conference. Norfolk(2013).

[13]Kong Bin, Ye Qiang, Chen Puhui. Post-buckling load transfer mechanisms of an integrated composite panel under uniaxial compression, J. Acta Materiae Compositae Sinica. 27,142(2010).

[14]Kong Bin, Ye Qiang, Chen Puhui. Post-buckling failure characterization of an integrated stiffened composite panel under uniaxial compression, J. Acta Materiae Compositae Sinica. 27,150(2010).

[15]Falzon B G, Steven G P. Buckling mode transition in hat-stiffened composite panels loaded in uniaxial compression, J. Composite Structures. 37,253(1997). 